\title{
IMPACTO DEL MEGAPROYECTO DE PUERTO NORTE EN LA TRANSFORMACIÓN URBANA DE SUS BARRIOS CIRCUNDANTES EN ROSARIO (ARGENTINA)
}

\section{IMPACT OF PUERTO NORTE MEGAPROJECT IN THE URBAN TRANSFORMATION OF ITS SURROUNDING NEIGHBORHOODS IN ROSARIO (ARGENTINA)}

Cintia Ariana Barenboim ${ }^{1}$

\section{Resumen}

La investigación aborda los efectos del gran proyecto urbano Puerto Norte, antiguamente predios del ferrocarril y el puerto, sobre los dos barrios tradicionales de su entorno: Refinería y Pichincha. Cabe señalar que si bien Puerto Norte es una centralidad en sí, todo el sector comienza a actuar, en algunas situaciones, en forma conjunta. Tal es el caso de las avenidas limítrofes donde se están construyendo edificios de similares características (tipología, usos, destinatarios).

La metodología se centra en un análisis de contenido de documentos escritos y gráficos como también en un abordaje descriptivo con el recorrido en los barrios. Los principales resultados evidencian que las transformaciones urbanas son desiguales, Pichincha tuvo un desarrollo edilicio anterior al inicio de sus obras y de mayor envergadura mientras que Refinería sufre un impacto menor pero directamente vinculado con Puerto Norte.

En este contexto, se introduce la temática de los grandes proyectos urbanos y se analiza la configuración histórica-urbana de todo el sector. Luego, se exponen los criterios de reordenamiento urbano, procesos de completamiento y densificación, junto a los cambios en los usos de suelo de ambos barrios durante la última década.

Palabras claves: gran proyecto urbano, impactos físicos-funcionales, transformaciones edilicias, barrios patrimoniales aledaños, Rosario.

\begin{abstract}
The research addresses the effects of large urban project Puerto Norte, formerly land of railways and the port, on the two traditional districts of its environment: Refineria and Pichincha. It should be noted that while Puerto Norte is a centrality itself, the entire sector starts to act in certain situations together. Such is the case of the neighboring streets where buildings are being built with similar characteristics (type, uses, and recipients).

The methodology focuses on a content analysis of written and graphic documents as well as a descriptive approach with the tour in the neighborhoods. The main results show that the urban transformations are unequal, Pichincha had a previous housing development at the beginning of his works and larger while Refineria suffered less impact but directly linked to Puerto Norte.

In this context, the issue of large urban projects are introduced and historical-urban settings across the sector is analyzed. Then, the criteria for urban renewal, completion and densification processes, together with changes in land use of both neighborhoods are exposed during the last decade.

Keywords: big urban project, physical - functional impacts, edilicial transformations, heritagesurrounding neighborhoods, Rosario.

\footnotetext{
1 Posdoctorado en segregación socioespacial y valoración inmobiliaria (CURDIUR-UNR). Investigadora Asistente del CONICET. Docente Titular de Planeamiento y Urbanismo (FCEIA-UNR), Enfoques Socioantropológico del Habitar, Análisis del Mercado Inmobiliario y Estrategias de Inversión (FA-UAI). Directora y co-directora de trabajos de investigación radicados en el Centro de Alto Estudios Arquitectura, Secretaria de Ciencia y Técnica y Lincoln Institute of Land Policy. E-mail: arq.barenboim@gmail.com
} 


\section{INTRODUCCIÓN}

Los grandes proyectos urbanos, como importantes obras emblemáticas en su integración con la ciudad, originan profundos impactos económicos, culturales, ambientales y urbanísticos (Lungo, 2004). Al respecto agrega Cuenya (2012) que producen al menos tres modificaciones claves en la materialización y funcionamiento de la ciudad: en lo físico-funcional, reproducen las condiciones de los centros urbanos pero como sitios exclusivos con edificios, servicios e infraestructura de alto nivel con múltiples usos; en la rentabilidad de los usos de suelo, dado que se produce un gran aumento en la valorización en los mismos predios y en las áreas aledañas y en los mecanismos de gestión pública, asumiendo el Estado un rol de promotor que exige un nuevo sustento normativo.

La presente investigación se orienta en la primera modificación, es decir, en los efectos físicos de los megaproyectos en el entorno de su implantación.

El área de estudio comprende dos situaciones distintas, coexistiendo fuertes dinámicas de transformación con componentes de valor urbano. Por un lado, la nueva centralidad de Puerto Norte, contigua al área central rosarina, garantizando la continuidad de los espacios públicos sobre el río Paraná y concentrando diversos establecimientos comerciales, residenciales y equipamientos. El sector está compuesto por siete unidades de gestión con diversas situaciones dominiales (propiedad público y propiedad privada), normativas y estados de construcción. Por el otro, dos tradicionales barrios aledaños al proyecto urbano, al noroeste Refinería ${ }^{2}$ y al sur Pichincha', que no habían sido alcanzados por el boom de la construcción de los '70, posicionándose en la actualidad como unas de las zonas de mayor potencialidad de crecimiento y demanda inmobiliaria (ver Plano $N^{\circ} 1$ ).

\footnotetext{
${ }^{2}$ Los límites del barrio Refinería (oficialmente Islas Malvinas) se ubican: Av. de las Tres Vías, Av. Francia, vías del Ferrocarril Central Argentino y Av. Alberdi.

${ }^{3}$ El barrio Pichincha se encuentra delimitado por: Bv. Oroño, Tucumán, Suipacha, Urquiza, Vera Mujica, Av. Del Valle y Rivadavia.
} 
Plano N 1: Localización del gran proyecto urbano Puerto Norte y los barrios aledaños Refinería y Pichincha en Rosario.

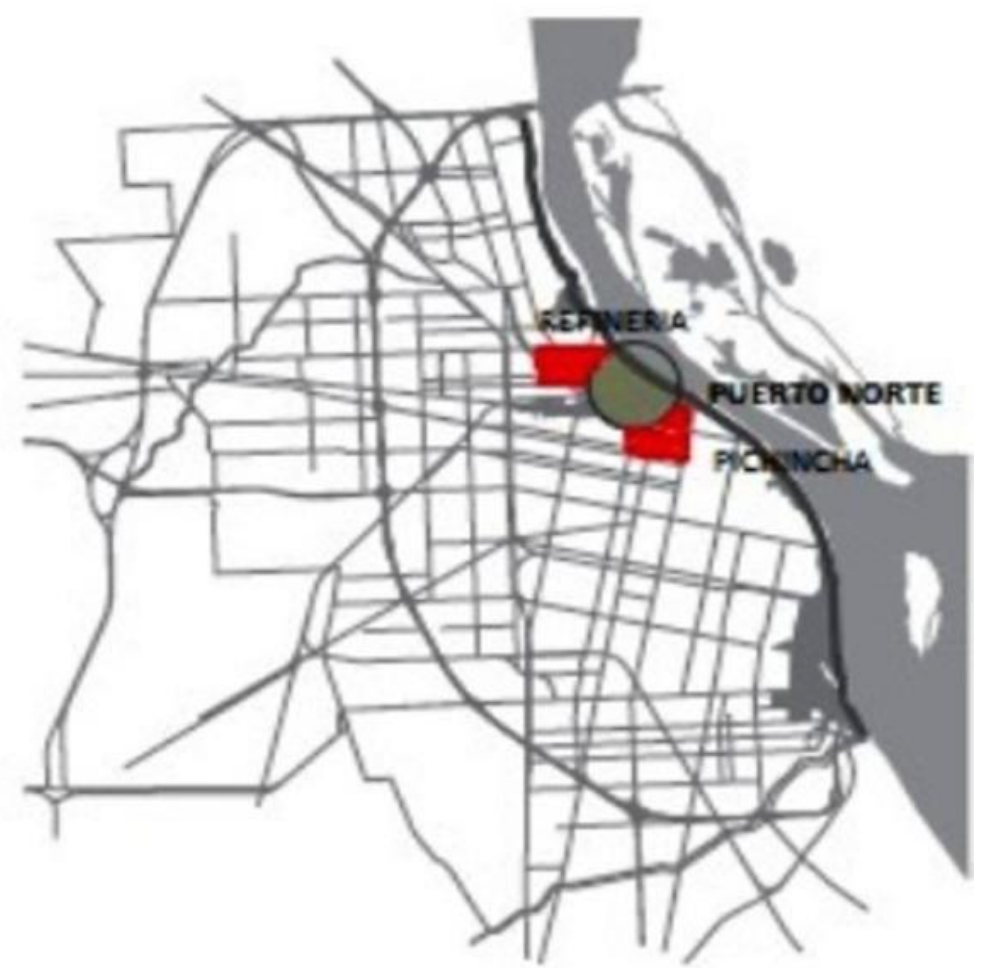

Fuente: Adaptado de la Dirección General del Plan Director, 2011.

\section{CONFIGURACIÓN HISTÓRICA-URBANA DEL ÁREA GENERAL (EX- ATRAVESAMIENTO NORTE)}

La reconstrucción del proceso de formación y crecimiento del sector brinda importantes elementos para el análisis de la configuración actual.

Un antecedente importante es el trabajo realizado por Martínez de San Vicente (1987) que evalúa el antigua área de Atravesamiento Norte, hoy macrocentro (incluye los barrios Puerto Norte, Refinería y Pichincha), entendida como área de ordenamiento prioritario. El sector presentaba tres rasgos fundamentales: el primero depende de las extensas instalaciones ferroviarias que lo definen, creando un delante y un detrás y restando continuidad al tejido urbano; el segundo guarda relación con el rol productivo de un fragmento del área: Refinería y el puerto anexo a ella; el tercero se refiere a la condición de patrimonio preciado y cada vez más escaso de la costa sobre el río. 
A mediados del siglo XIX, las empresas ferroviarias que comenzaron a instalarse en la ciudad privilegiaron el área como vía de acceso a la costa y el puerto. De esta manera, se fue produciendo en la zona una concentración de instalaciones que significó una interrupción urbana, quedando el norte y noroeste segregado, sumado a las líneas de tranway y tranvías que servían las estaciones. Por su parte, el río impuso un límite natural generando el sitio adecuado para las instalaciones portuarias a lo largo de la costa central (Martínez de San Vicente,1987).

Las urbanizaciones alrededor de las instalaciones ferro-portuarias (hoy Puerto Norte) se concretaron en distintos momentos a través de numerosas operaciones y magnitudes.

En un primer momento, al sur comienza la formación del barrio Pichincha, cuyo tejido nunca tuvo rasgos particularizantes. La lógica que se impuso fue la expansión del área central de la ciudad, organizada a lo largo de ciertos ejes viarios, con una cierta homogeneidad en la dimensión de las manzanas. La dominancia funcional estaba vinculada a usos residenciales, productivos y comerciales. También era la zona prostibularia reglamentada de la ciudad. ${ }^{4}$

En un según orden, al noroeste nace signado por la condición de marginalidad así como por la actividad portuaria el barrio Refinería. La no continuidad vial con el centro provocó que el trazado del área tomara las direcciones de las propiedades rurales, resultando una gran variedad de tipos de manzanas. Posteriormente, se constituyeron puntos de vinculación con el centro, perdiendo su condición original de periferia. ${ }^{5}$ El sector se define con actividades productivas siendo el saladero y la refinería de azúcar los edificios fundacionales a partir de los cuales se establecen las primeras construcciones orientadas a los obreros.

Tanto en Pichincha como en Refinería se desarrolló un proceso de progresiva expansión de la planta urbana hacia el oeste, presentando marcadas discontinuidades. Los lotes de distintas dimensiones se alternaban entre grandes superficies de usos productivos (ancho entre $27 \mathrm{~m}$ y 36 m) y lotes urbanos (ancho de $9 \mathrm{~m}$ a $11 \mathrm{~m}$ ).

El crecimiento del sector fue también consecuencia de la gran inmigración extranjera que ingresaba al país y de las migraciones internas ocasionadas por la gran oferta laboral que ofrecía el

\footnotetext{
${ }^{4}$ El desarrollo demográfico y comercial que experimentaba Rosario y su ubicación geográfica, propiciaron la instalación de prostíbulos. Entre 1874 y 1932 aparecieron una serie de ordenanzas que regulaban su ejercicio. El sistema se conoció como prostitución reglamentada que combinaba aspectos sanitarios, políticos, administrativos y policiales. Sin embargo, muchos se desempeñaban en la clandestinidad por lo que el municipio intentó controlar y delimitar una zona para su funcionamiento (Dócola y Múgica, 2006).

${ }^{5}$ La habilitación del pasaje Celedonio Escalada a principios de siglo y luego el camino Nuevo a San Lorenzo (Av. Alberdi) constituyeron los únicos puntos de atravesamiento del área ferroviaria y de vinculación con el área central hasta la construcción, en la década del '70, del viaducto Avellaneda.
} 
puerto y el ferrocarril. Rosario en veinte años triplicó su población, multiplicó diez veces su comercio y el puerto ya ocupaba el segundo lugar en el mundo por el monto de sus operaciones de grano (Barenboim, 2011).

Durante 1930 a 1980 se continúo un constante proceso de completamiento de densidades medias y posteriormente se incorporaron modificaciones y ciertas sustituciones. Al mismo tiempo, debido a la presión por el incremento de la renta urbana, los lotes sufrieron procesos de fragmentación y reducción de la superficie interna. Martínez de San Vicente (1987) menciona que los procesos de subdivisión asumieron en las manzanas tres modalidades:

Disminución de la dimensión de la manzana y aparición de pasajes que la seccionan en dos permitiendo un incremento en la cantidad de lotes; subdivisión del lote frentista hasta alcanzar el ancho mínimo admisible y por último, la aparición de uno as más lotes internos como subdivisión del lote angosto y profundo" (Martínez de San Vicente, 1987, p.70).

Los lotes internos comprenden la división en propiedad horizontal de los emprendimientos conocidos como departamentos de pasillos que se van subdividiendo a partir de los años sesenta. El barrio Refinería (24\%) tuvo mayores subdivisiones que Pichincha (19\%), denotando este parcelamiento una indudable tendencia residencial.

Cabe señalar que el boom de la construcción de los años '70 no favoreció a estos dos barrios obreros y de casas modestas. ${ }^{6}$ Los emprendimientos estuvieron orientados a las clases medias localizados en distintos puntos de la ciudad, principalmente en el área central y bajo la modalidad de edificación en altura.

Asimismo, el puerto dejó de ser el motor de la ciudad y la población comenzó a reclamar la integración de la trama urbana con el rio. El Estado delineó dos planes que influyen sobre el sector. El Plan Regulador (1968) estableció el cese de la actividad portuaria en la ribera central y propuso Centros Urbanos, entre ellos el Scalabrini Ortiz. El Plan Regulador del Puerto (1970) concentró todas las actividades portuarias al sur y planteó recuperar un amplio frente urbano, posibilitando la parquización de espacios libres. Sin embargo, la tenencia de la tierra e instalaciones propuestas en los planes no se realizaron debido a los sucesivos cambios políticos.

\footnotetext{
${ }^{6}$ En los '70, la construcción era impulsada por las desgravaciones impositivas dispuestas por el gobierno. Los montos invertidos en inmuebles a construir gozaban de importantes ventajas. Las reducciones variaban según el tipo de propiedad; si era de bajo nivel, correspondía un $80 \%$, si era de mejor calidad, un $50 \%$. Para las empresas, fue muy rentable construir sin embargo no fueron los únicos actores; también las familias y los inversores edificaban. Los proyectos estaban dirigidos a los sectores de clase media. La ley determinaba características financieras, puesto que había un déficit habitacional (Barenboim, 2011).
} 
En las últimas décadas del siglo XX, se comienzan a implementar políticas públicas de ordenamiento territorial sostenidas por un modelo neoliberal. El gobierno se asocia con el sector privado, influyendo sus intereses económicos en las intervenciones urbanas.

El proceso de crecimiento en los barrios históricos de Pichincha y Refinería fue disminuyendo y siendo reemplazado por otro de degradación edilicia y urbana. La magnitud de la oferta de viviendas desocupadas produjo una baja en el costo de los inmuebles, siendo el precio de venta similar al de construcción.

Puerto Norte se presentaba como un área de oportunidad debido a su localización en el interior de la planta urbana sobre la costa, uniendo finalmente el norte con el centro y recuperando el patrimonio industrial local. Esto motivó que en 1991 el Plan Director establezca la transformación de los usos originales de Puerto Norte y que la Municipalidad junto con la Universidad Nacional de Rosario realice un Seminario Internacional de Proyectos Urbanos. En el 2004, el Municipio y el Colegio de Arquitectos de Rosario hicieron un llamado a Concurso Nacional de Ideas y Anteproyectos solicitando la propuesta del gran proyecto urbano.

Finalmente, la reconversión del antiguo puerto se establece con la gran operación público privada denominada "Centro de Renovación Urbana Scalabrini Ortiz", llevada a cabo en dos fases:

1. Primera Fase: define el sector a intervenir, la prolongación y ensanche de trazados, los nuevos usos, la condiciones de edificabilidad y los edificios de valor patrimonial a preservar del área de Talleres (Ordenanza N 6.271/96). El proyecto comprende el Parque Scalabrini Ortiz, el Shopping Alto Rosario y el supermercado COTO inaugurados en el 2004. Dichos establecimientos potencializan el desarrollo de Refinería, particularmente sobre la calle Junín, produciendo un gran impacto en la valorización inmobiliaria para uso comercial y residencial. Los efectos se expanden hacia el interior del barrio, provocando grandes sustituciones edilicias. En el mismo año el barrio Pichincha es declarado de "interés urbanístico", revalorizándose y adquiriendo un carácter patrimonial a nivel local.

2. Segunda Fase: define finalmente los trazados públicos, los espacios verdes y el esquema de densidades ordenada mediante la redacción de un Plan Especial para Puerto Norte (Ordenanza № 7.892/05). El sector se divide en siete Unidades de Gestión con menores superficies para agilizar y articular la implementación del proyecto. ${ }^{7}$ Este modelo impulsa

\footnotetext{
${ }^{7}$ Características de las siete Unidades de Gestión (Plan Urbano Rosario 2007 - 2017, 2011):

Unidad de Gestión 1: el predio de 8,4 has. Perteneciente a Servicios Portuarios S. A. contempla un conjunto residencial y comercial llamado. Metra iniciado en el 2014.
} 
una continua apropiación pública de los terrenos portuarios y facilita las inversiones inmobiliarias (infraestructurales y edilicias). Posteriormente, para cada unidad se realiza un plan de detalle, con sus propios indicadores urbanos, y un convenio urbanístico. La norma redujo los indicadores urbanos en el interior de Refinería con el fin de preservar el perfil histórico del barrio. Solamente se mantienen altos indicies edilicios sobre los corredores perimetrales, como por ejemplo la Av. Cándido Carballo (ver Plano №2).

Asimismo, el barrio Pichincha que ya venía reactivándose con el desarrollo de nuevos usos vinculados a su rol patrimonial, cultural y turístico, a partir de la restauración de edificios históricos, y residenciales con el completamiento de edificaciones de bajas y altas densidades, comienza a ser impactado por Puerto Norte. Esto se evidencia en la renovación total en la Avdas. Limítrofes de Rivadavia y Del Valle. Cabe señalar que la sustitución edilicia fue favorecida también con una normativa particular sobre el barrio Pichincha (Ordenanza N8.125/06).

Unidad de Gestión 2 - Sector 1 Forum: el terreno de una sup. de 2,1 has. adquirido por la empresa TGLT para desarrollar un emprendimiento residencial entre el 2008 y 2013.

Unidad de Gestión 2 - Sector 2 Ciudad Ribera: la superficie es de 2,1 has. propiedad de INGECONSER S.A., propone: viviendas, oficinas, hotel y centro de convenciones, iniciado en el 2009 y finalizado en el 2014.

Unidad de Gestión 4: la superficie de 2,3 has. de distintos inversores locales para uso residencial todavía no ha comenzado las obras.

Unidad de Gestión 5: el sector de 1,7 has. desarrollado por Inversiones y Mandatos S. A. está compuesto de tres unidades: conjuntos residenciales Dolfines Guaraní y Torre Embarcadero y edificio Nordlink, destinado a oficinas Premium, iniciado en el 2005 e inaugurado en el 2010.

Unidad de Gestión 6: el predio de 2,5 has. perteneciente a Servicios Portuarios S. A. proyecta el complejo Maui para viviendas y un hotel internacional, iniciado en el 2009.

Unidad de Gestión 3 y 7: los terrenos nacionales del Administrador de Infraestructuras Ferroviarias, superficies de 36,2 has. y 20,7 has. no han iniciado aún las obras.

Manzana 407: la superficie de 5 has. cuyo dominio es de FUNDAR y ROSENTAL destinado a uso residencial y comercial, comenzó en el 2007 y continúa en obra. 
Plano N²: Fases del Centro de Renovación Urbana Raúl Scalabrini Ortiz, Unidades de Gestión y corredores perimetrales.

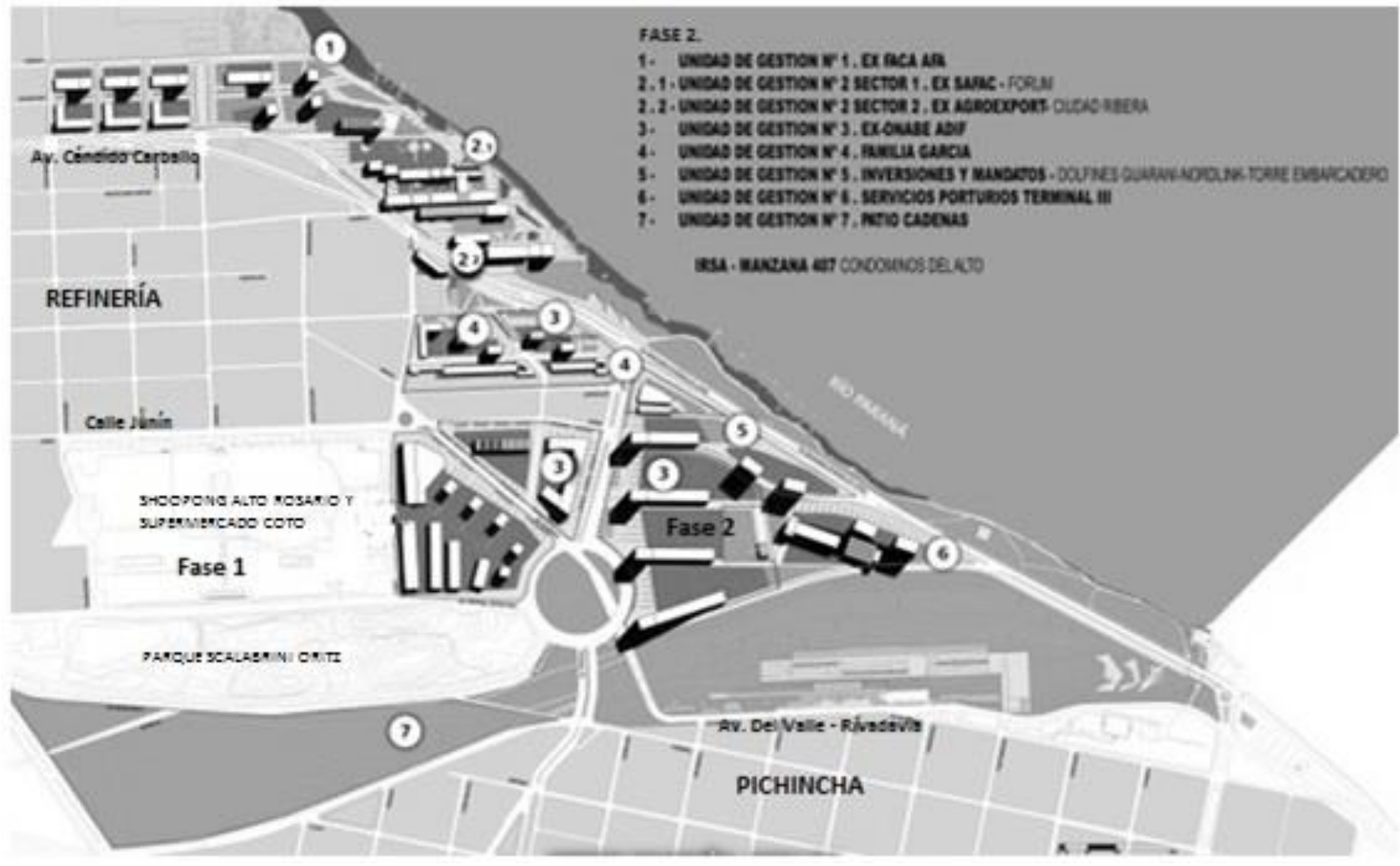

Fuente: Adaptado de la Dirección General del Plan Director, 2011.

\section{CRITERIO DE REORDENAMIENTO URBANO DEL ENTORNO DE SU IMPLANTACIÓN}

A partir del año 2005, con la finalización de la fase 1 Área Talleres y el comienzo de las obras en la fase 2 Puerto Norte (Unidad de Gestión 5) del Centro de Renovación Urbana Scalabrini Ortiz, los barrios Pichincha y Refinería comienzan a sufrir cambios significativos en su configuración física-funcional. También el perfil social del entorno de su implantación fue cambiando dado que el gran proyecto central, destinado a las empresas privadas y los sectores sociales de altos ingresos, lentamente fue encareciendo la vida urbana y expulsando a ciertos pobladores originales (ex-obreros industriales y ferro-portuarios). La población se incremento según los Censos Nacionales de Población y Vivienda (2001 y 2010): Refinería, 4.308 y 4.550 habitantes; Pichincha, 9.290 y 10.528 habitantes, respectivamente.

En estos procesos el capital inmobiliario orientado por las políticas locales, a través de los distintos planes especiales $u$ otras normas que modifiquen los indicadores urbanos, tiene un gran protagonismo. Igualmente, Rosario estaba transitando un momento económico favorable y un 
gran desarrollo de la construcción. La inversión inmobiliaria que anteriormente se contenía en el área central se fue extendiendo por la zona contigua, la segunda ronda de bulevares, donde se ubica Pichincha, y la tercera, donde esta Refinería. Estos barrios poseen una posición estratégica, debido a su emplazamiento tan cercano a la ribera, y forman parte de un proceso de renovación edilicia que tiene lugar actualmente en el borde de la ciudad.

El criterio de reordenamiento en los sectores aledaños a Puerto Norte tenía dos propósitos esenciales: la protección de sus particulares condiciones urbanas y la renovación y mejoramiento edilicia. En consecuencia, se reglamentan alturas máximas, factor ocupacional suelo, usos permitidos, modificación en la línea municipal, preservación de edificios, entre otros indicadores a través del Plan Especial barrio Pichincha (Ordenanza N 8.125/06) y el Reordenamiento Urbanístico Segundo Anillo Perimetral donde esta Refinería (Ordenanza № 8.980/12).

Los corredores urbanos son inducidos a una profunda sustitución edilicia debido a su deterioro, obsolescencia de las instalaciones y la inconveniencia de la persistencia de determinados usos. La intención es que se comiencen a construir edificios de similares características que en Puerto Norte. Tal es el caso de la Av. Del Valle - Rivadavia, denominado Frente de Renovación Urbana, donde se permiten la construcción de torres de gran altura, triplicando los índices anteriores (Ordenanza $N^{\circ}$ 8.125/06) y la Av. Cándido Carballo implementa un plan especial (Ordenanza $\mathrm{N}^{\circ}$ 7.956/10) donde se propone una altura en concordancia con los prototipos edilicios correspondientes al Plan de Detalle de la Unidad de Gestión 1 Metra. ${ }^{8}$ Posteriormente, el Reordenamiento Urbanístico Segundo Anillo Perimetral modificó los indicadores de los corredores urbanos secundarios unificando las alturas de Junín y Thedy. Si bien esta última calle incrementa su altura, Junín la reduce en relación a lo permitido en la normativa anterior (Ordenanza № $7.892 / 05) \cdot{ }^{9}$

Los usos admitidos están relacionados con actividades residenciales y comerciales, administrativas y de servicios a la comunidad. Las normas eliminan las industrias, depósitos y cualquier otra actividad que sea contaminante e incompatible con el predominante uso residencial del sector.

\footnotetext{
${ }^{8}$ La altura permitida en Pichincha pare el Frente de Renovación Urbana (sector 1: $36 \mathrm{~m}$ y sector 2: $66 \mathrm{~m}$ ) es considerablemente mayor en relación a la de la Av. Carballo (sector 1: $15 \mathrm{~m}$ y sector 2: $25 \mathrm{~m}$ ).

${ }^{9}$ La Ordenanza no 7.892 establecía para la calle Junín, ubicada frente al Shopping Alto Rosario, una altura máxima superior que la actual normativa (se reduce de $30 \mathrm{~m}$ a $19 \mathrm{~m}$ ).
} 


\section{PROCESOS DE DENSIFICACIÓN Y COMPLETAMIENTO DEL TEJIDO}

Las transformaciones físicas de la estructura parcelaria se produce de dos maneras en los barrios aledaños a Puerto Norte: rehabilitación de edificios patrimoniales y la construcción de obras nuevas o ampliaciones.

La rehabilitación de algunos edificios de carácter histórico permitió conservar gran parte de la estructura edilicia de la época, pero no las funciones debido a que habían cambiado sus características originales. El proceso se dio más fuertemente en Pichincha que en Refinería, dado que cuenta con cuatro áreas de protección histórica. ${ }^{10}$

La construcción que se desarrolla en dichos barrios produce procesos de completamiento y renovación del tejido. El completamiento se da en el interior de las áreas a través de obras de ampliación o reforma. Este tipo de intervención se desarrolla más intensamente y en todo el sector en Pichincha $\left(18.442 \mathrm{~m}^{2}\right)$ que en Refinería $\left(1.361 \mathrm{~m}^{2}\right)$ donde se manifiesta en algunas calles, durante el periodo de estudio. Las nuevas construcciones son intensas en ambos barrios y están localizadas principalmente en los corredores urbanos (78.794 $\mathrm{m}^{2}$ y $21.290 \mathrm{~m}^{2}$, respectivamente).

Los procesos de renovación traen aparejados la sustitución de tipologías edilicias, generalmente casas o galpones antiguos en desuso. Entre los años 2005 y 2006 las demoliciones han sido muy intensivas en todo Pichincha dado que la normativa lo permitía. Esto produce una gran diferencia con Refinería que se incrementaron en los últimos años (16.012 m² y $2.274 \mathrm{~m}^{2}$ respectivamente). En ambos casos se involucran parcelas contiguas, orientadas a emprendimientos de mayor envergadura.

\footnotetext{
10 Las áreas de protección histórica en el barrio Pichincha son: Ámbito Histórico Pichincha, Corredor Urbano Av. Salta, Entorno Casa del Estudiante y Calle Jujuy 2200-2300.
} 
Gráfico N 1: Tipos de operación según barrios Pichincha y Refinería. Período 2005 - 2014.

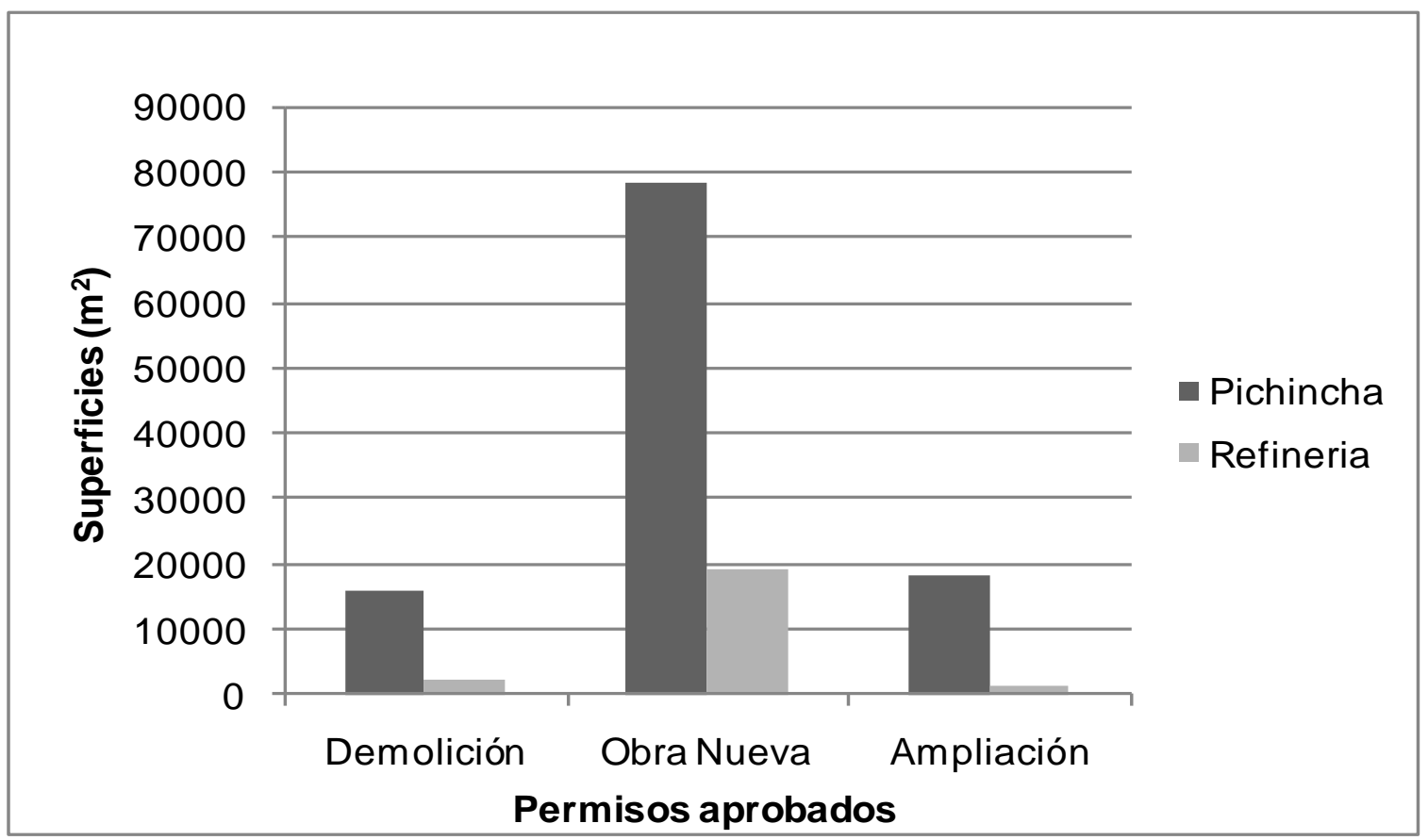

Fuente: Elaboración propia con datos de los permisos aprobados en la Dirección de Obras Particulares, 2015.

La evolución de los permisos de construcción, entre los años 2005 y 2014, varían en cada barrio según el tipo de operación: demolición, ampliación u obra nueva. Asimismo, dentro de ellos, en los principales corredores urbanos se desarrollan las dinámicas más intensas de la construcción (ver Fotografías $N^{\circ} 1,2$ y 3). Cabe señalar que se encuentran además presentados en la Dirección General de Obras Particulares y/o Programa de Actualización Normativa (proyectos con condiciones especiales), expedientes de edificación y demolición que todavía no han obtenido los permisos correspondientes. 
Fotografías $N^{\circ} 1,2$ y 3: Edificios de viviendas en los corredores Junín, Cándido Carballo y Del Valle Rivadavia.

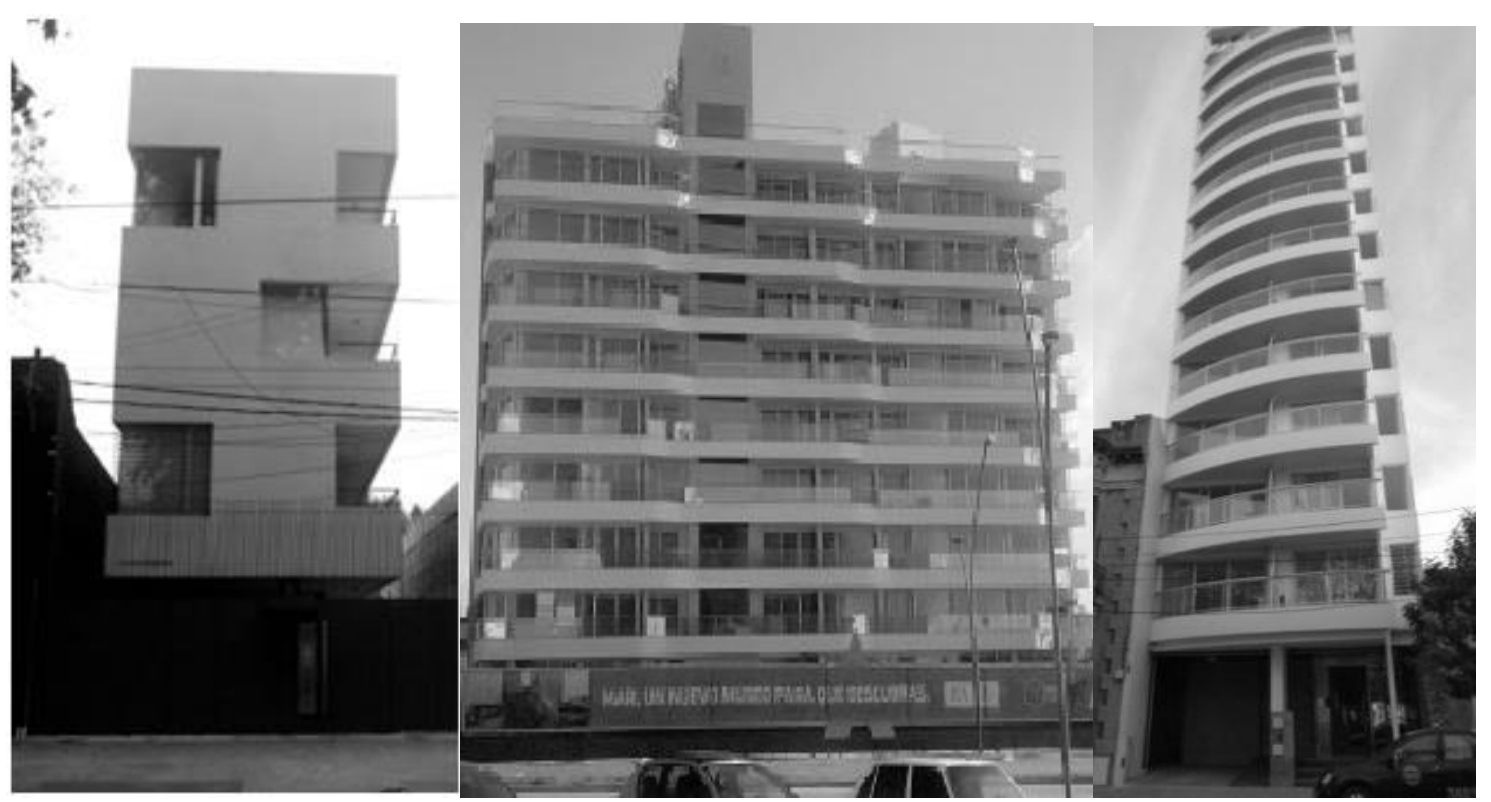

Fuente: tomadas por la autora, 2014.

Pichincha sobrelleva demoliciones más intensas y distribuidas en todo el barrio hasta el año 2006. Luego entre 2007 y 2009 se reducen a la mitad, finalmente a partir de allí se vuelven a reducir a la mitad, orientándose generalmente en el centro y primeras cuadras de la Av. Del Valle Rivadavia.

Las obras de edificación también tienen su auge hasta el 2008, momento donde se reducen considerablemente pero manteniendo las superficies constantes hasta el 2014. Dentro del corredor urbano se consolidan los edificios nuevos en las cuadras centrales. Por último, las ampliaciones son importantes en todo el barrio (ver Gráfico $N^{\circ} 2$ ). 
Gráfico N² 2: Evolución por año de tipos de operación en el barrio Pichincha.

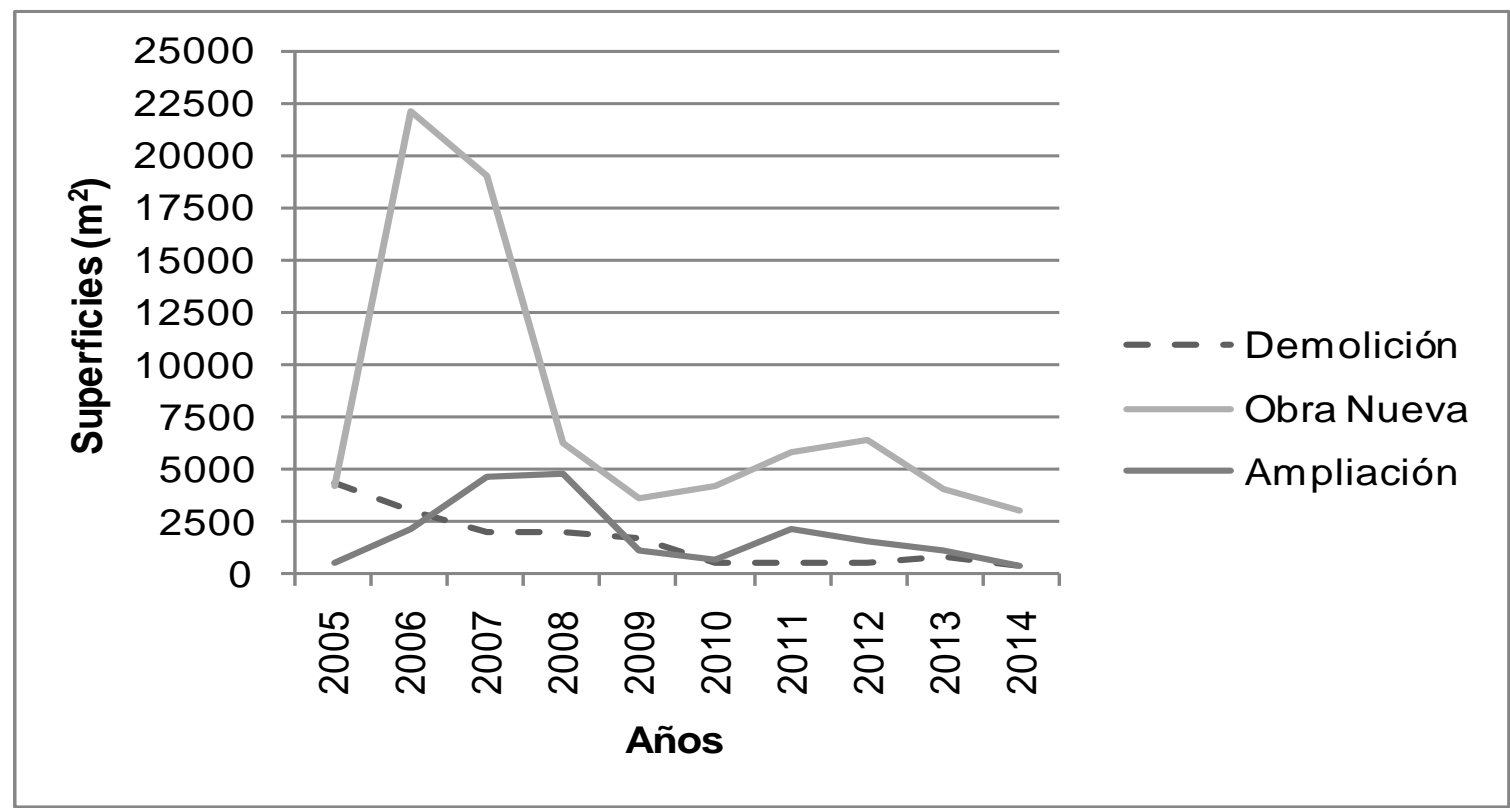

Fuente: Elaboración propia con datos de los permisos aprobados en la Dirección de Obras Particulares, 2015.

Refinería comienza a contar con pequeñas demoliciones a partir del año 2007, intensificándose desde el 2010 al 2014 a lo largo de toda la Av. Cándido Carballo.

Las obras nuevas se incrementan desde el 2006 (un año después de aprobada la Ordenanza № 7.892/05) a la actualidad. Las superficies se dividen en los incipientes edificios sobre la Av. Cándido Carballo, viviendas en su interior y grandes comercios en las calles Junín y Vélez Sarsfield. Por último, las ampliaciones son escasas, manteniendo los mismos valores, distribuidas en todo el barrio. 
Gráfico $n^{\circ}$ 3: Evolución por año de tipos de operación en el barrio Refinería.



Fuente: Elaboración propia con datos de los permisos aprobados en la Dirección de Obras Particulares, 2015.

Los principales corredores urbanos si bien cuentan con ciertos edificios nuevos todavía se caracterizan por la presencia dominante de construcciones bajas (Pichincha 80 \% y Refinería $94 \%$ ).

El relevamiento realizado constató que los lotes frentistas son 120, distribuyéndose de forma distinta las alturas durante el período 2005-2014. El proceso de renovación urbano se inicia en el 2006, contando con más lotes, de mayores dimensiones y en un recorrido más extenso en la Av. Del Valle - Rivadavia mientras que en la Av. Cándido Carballo fue recién en el año 2011, con menor cantidad de lotes de pequeña superficie (ver Tabla $N^{\circ} 1$ ).

Actualmente el proceso de renovación urbana no está terminado contando con 10 y 5 lotes baldíos (Cándido Carballo y Del Valle - Rivadavia, respectivamente) donde no están definidos los proyectos edilicios. Se estima que será más intensa la renovación en el corredor de Refinería.

Tabla N 1: Relación de alturas con la ocupación de lotes en los Corredores Urbanos en el año 2014.

\begin{tabular}{|c|c|c|c|c|}
\hline Corredores Urbanos & \multicolumn{2}{|c|}{ Del Valle - Rivadavia } & \multicolumn{2}{c|}{ Cándido Carballo } \\
\hline Niveles & Cantidad & Porcentaje & Cantidad & Porcentaje \\
\hline PB +13 & 8 & $12 \%$ & 0 & 0 \\
\hline
\end{tabular}




\begin{tabular}{|c|c|c|c|c|}
\hline PB +10 & 3 & $4 \%$ & 0 & 0 \\
\hline PB +8 & 0 & 0 & 1 & $2 \%$ \\
\hline$P B+4$ & 0 & 0 & $2 *$ & $4 \%$ \\
\hline PB + & 3 & $4 \%$ & 0 & 0 \\
\hline PB + & 4 & $6 \%$ & 3 & $6 \%$ \\
\hline PB/ PB +1 & 52 & $74 \%$ & 44 & $88 \%$ \\
\hline Total lotes & 70 & $100 \%$ & 50 & $100 \%$ \\
\hline
\end{tabular}

Fuente: Elaboración propia con datos de relevamiento en corredores urbanos, 2015.

La calle Junín no se analizo dado que la normativa no induce la edificación en altura, contando toda la calle con edificaciones de planta baja y planta baja y un piso. Solamente hay un edificio de planta baja y tres pisos (ver Fotografía $N^{\circ} 1$ ).

\section{TRANSFORMACIÓN E INCREMENTO DE NUEVAS ACTIVIDADES}

Junto al completamiento y la renovación de las edificaciones comienzan a parecer nuevos usos en las ex-áreas industriales del entorno de implantación de Puerto Norte. A pesar de que ambos barrios son de carácter patrimonial los roles que adquieren, el momento donde se registran los cambios, la localización y el tipo de actividad de los mismos es desigual en cada uno de ellos, teniendo un perfil más turístico - comercial Pichincha. Especialmente los corredores urbanos de los barrios adquieren múltiples características a lo largo de su trayecto siendo los sitios donde más se evidencia el impacto del gran proyecto urbano de Puerto Norte (ver gráfico $\left.n^{\circ} 4\right)$. 
Gráfico N 4: Comparación de los usos de suelo en los barrios de Pichincha y Refinería de Rosario.

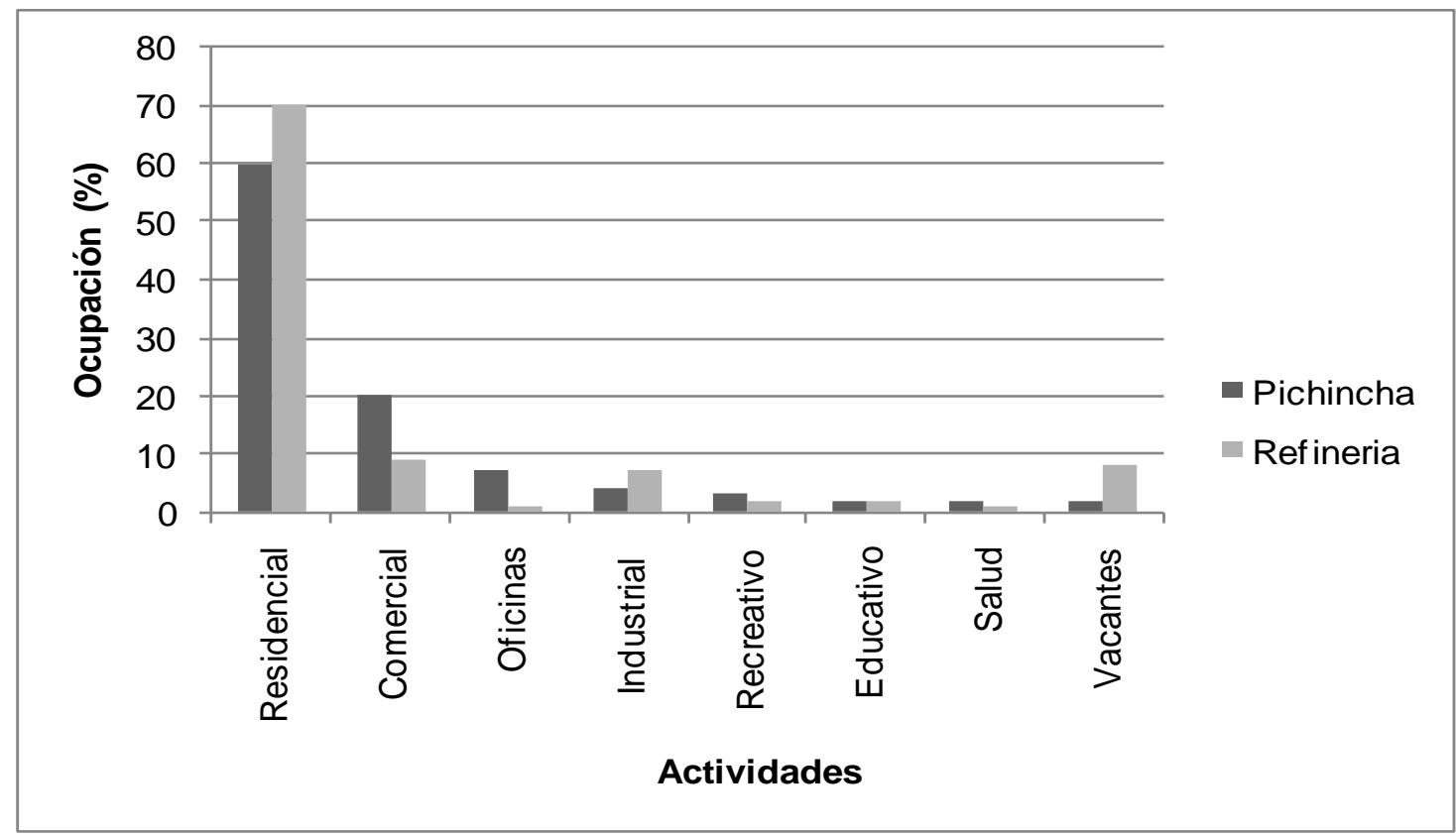

Fuente: elaboración propia en base a relevamiento in situ de lotes del sector, 2015.

Pichincha tiene una imagen que podríamos denominar pintoresca, con una importante dinámica residencial y comercial en relación a la ciudad. A partir del año 2003, el sitio comienza su período de mayor reactivación y se fomentan importantes inversiones que continúan desarrollándose hasta la actualidad.

Las viviendas están orientadas a clases sociales medias y medias - altas, de carácter colectivo (edificios en altura y departamentos de pasillo) o individual, representando el $60 \%$ de los lotes.

Los otros usos que aparecen se relacionan con su nuevo rol turístico - cultural, respondiendo a iniciativas públicas y privadas. Las primeras comprenden la Secretaria de Cultura, la Feria de Artesanías del Boulevard, el Mercado de antigüedades Feria Retro, el Mercado Solidario y la Asociación Vecinal (3\%) localizadas sobre el límite norte del barrio, contiguo a Puerto Norte. Las segundas son los comercios minoristas dedicados a la venta y restauración de antigüedades, bares, restaurantes, boutiques de diseñadores, ferias de diseño de moda, arte y decoración, boliches, pubs, alojamientos (20\%), las pequeñas oficinas ( $8 \%$ ) y los escasos talleres y depósitos (4\%) ubicados en el interior del barrio, con mayor concentración en las calles principales y el sector norte sobre la costa. 
La actividad industrial fue prohibida a partir del año 2007, con el Plan Especial barrio Pichincha (Ordenanza № 8.125/06), teniendo como intención cambiar la funcionalidad del sector. Esto produjo que se incorporen al barrio lotes vacantes de grandes dimensiones para el desarrollo de nuevos usos.

Los establecimientos de servicio a la comunidad son educativos, deportivos, clínicas y consultorios (7\%).

Particularmente a lo largo de la Avenida Del Valle - Rivadavia se van registrando cambios de actividades entre los años 2005 y 2014. En su comienzo quedan algunos galpones abandonados y/o en demolición para el futuro desarrollo de emprendimientos inmobiliarios orientados a viviendas de alta gama y oficinas premium. En seguida se establecen actividades comerciales relacionadas al entretenimiento nocturno (bares, restaurantes y boliches) entremezcladas con edificios de vivienda, en mayor grado, y de pequeñas oficinas (inmobiliarias, estudios de arquitectura, jurídicos y contables, sedes administrativas de empresas, etc.), en menor orden. Por último, se consolida un frente totalmente residencial.

Fotografías N 4 y 5: Restaurante Rolling Road y boliche Borggea sobre la Av. Del Valle - Rivadavia.

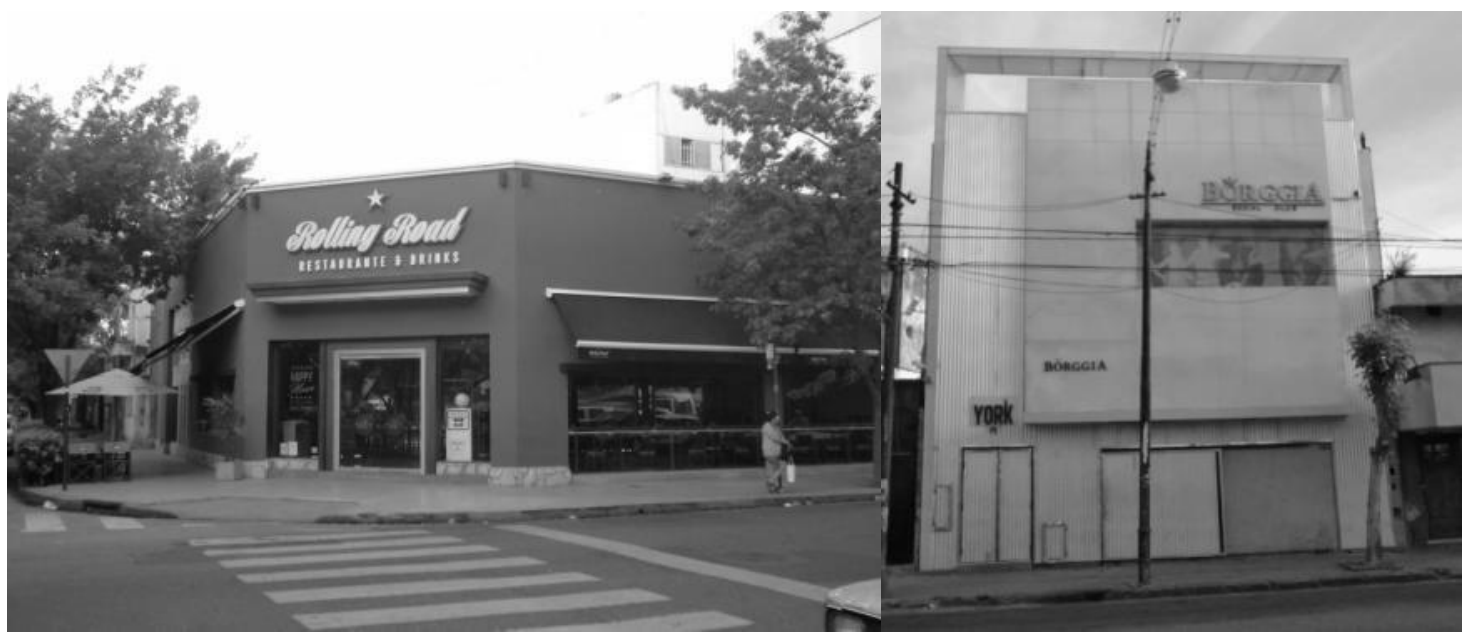

Fuente: tomadas por la autora, 2014.

Refinería tiene un carácter más residencial orientado a una clase social media y media baja con cierta actividad comercial minorista, de abastecimiento del barrio, e industrias locales. Los cambios comienzan a registrarse en el año 2005 luego de la construcción del Shopping Alto Rosario y el supermercado COTO sobre la calle Junín expandiéndose hacia el interior del barrio. Posteriormente en el año 2011 con la apertura de la avenida Luis Cándido Carballo y el desarrollo 
de la Unidad de Gestión 1 Metra comienza a provocarse grandes sustituciones edilicias en dicho corredor.

Las viviendas constituyen el $70 \%$ de los lotes y son mayoritariamente unifamiliares, comenzando a realizarse edificios en altura sobre las calles principales y avenidas recién en los últimos años. Asimismo, en la futura Unidad de Gestión 3 Ex- Onabe se encuentra el asentamiento irregular Villa Margarita.

Los comercios de jerarquía son concesionarias automotrices de alta gama, restaurant y empresa de servicios de catering ubicadas frente al Shopping y los locales de menor orden como mercados, verdulerías, bares, casas de electricidad, venta de ropa, librerías, se distribuyen en el interior del barrio (9\%).

La actividad industrial lentamente va disminuyendo y siendo sustituida por otros usos, quedando todavía en funcionamiento algunos depósitos y talleres de grandes dimensiones (7 \%).

Los equipamientos urbanos contribuyen a una mejor calidad de vida para los habitantes de la zona incluyendo jardines de infantes, escuelas, clubes, centros de salud y el Centro Cultural Ciudad de los Niños junto a la plaza del Mutualismo sobre la costa (5\%). El edificio de seguridad, constituido por la cárcel de Mujeres, se presenta en algunas ocasiones como un uso conflictivo con el uso residencial dominante.

El barrio actualmente está en un proceso de reconversión de usos y servicios generando grandes transformaciones edilicias en las instalaciones existentes. Sin embargo aún queda un gran porcentaje de terrenos e inmuebles vacantes de uso (8\%).

Los corredores urbanos presentan diversas situaciones según su escala en Refinería:

La calle Junín, comienza un proceso de reconversión de usos a partir de la instalación del Shopping Alto Rosario y el Supermercado Coto en el 2004. En las primeras cuadras hay restaurantes y locales barriales (peluquería, autoservicio, etc.) entremezcladas con viviendas, luego hay dos cuadras donde se localizan varias concesionarias automotrices y una empresa de servicios de catering y culminando viviendas residenciales (frente al Shopping).

La avenida Cándido Carballo registra cambios en las actividades a partir del año 2011, con la instalación de la concesionaria en su extremo frente a un antiguo galpón. En seguida se encuentran entremezcladas viviendas con algunos pequeños comercios (vivero, taller mecánico, salón de ventas) y edificaciones en demolición, para el futuro desarrollo de emprendimientos inmobiliarios, finalizando con el Centro Cultural. 
Fotografías $N^{\circ} 6$ y 7: Concesionaria automotriz Mercedes - Benz en calle Junín y Centro Cultural Ciudad de los Niños sobre la Av. Cándido Carballo.

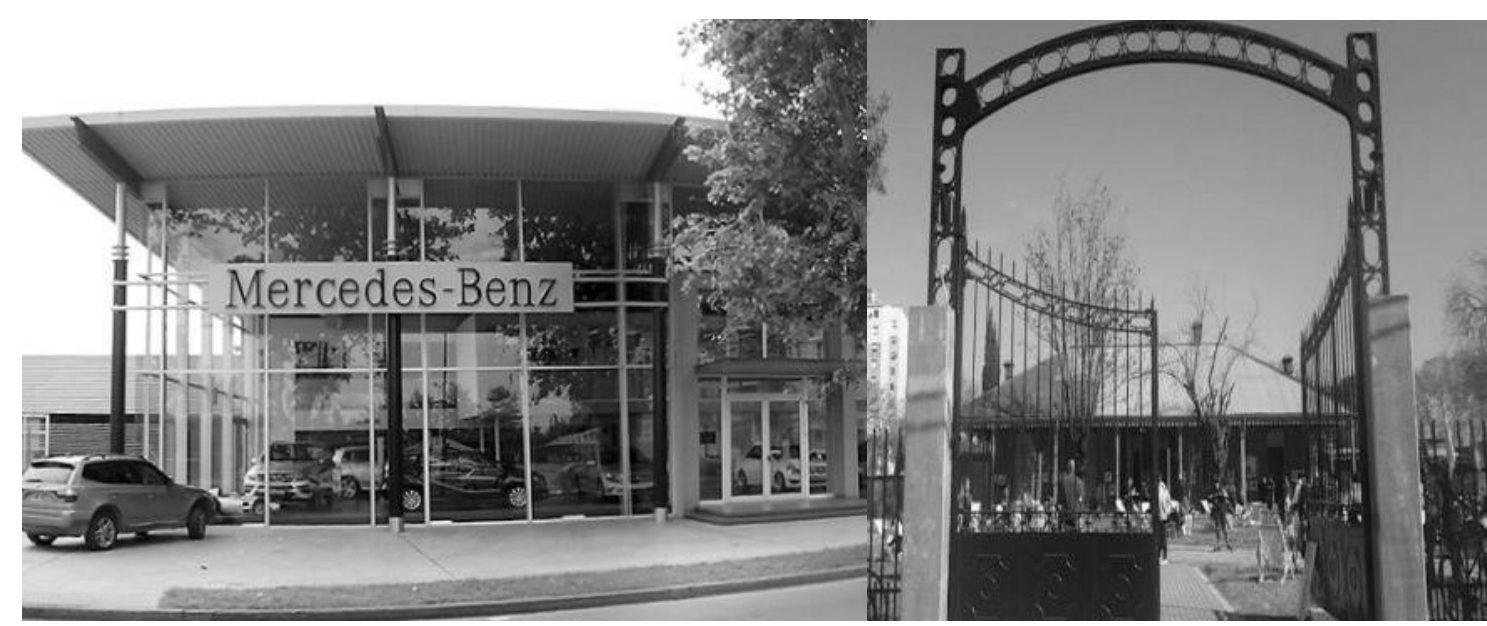

Fuente: tomadas por la autora, 2014.

\section{CONCLUSIONES}

Puerto Norte se propone como una nueva centralidad en un lugar estratégico, donde se encontraban las antiguas y abandonadas instalaciones ferro-portuarias de la ciudad de Rosario. Este se destina a residencias, comercios y oficinas con infraestructura y servicios de alto nivel, dando respuestas a nuevos sectores sociales.

Actualmente, el gran proyecto urbano no está concluido habiéndose edificado el $20 \%$ de la superficie total. Falta que se inicien las obras de la Unidad 4, completen las edificaciones en curso (Unidad 1, 6 y Manzana 407) y aprueben los planes de detalle en los amplios terrenos nacionales (Unidad 3 y 7). Sin embargo, desde su inicio en el año 2005 pueden reconocerse ciertas transformaciones urbanas en el entorno de su implantación (cambios de usos, densificación de tejidos existentes sobre avenidas y completamiento de vacíos interiores).

Al sur de Puerto Norte, el barrio Pichincha tuvo un desarrollo edilicio anterior al inicio de sus obras y de mayor envergadura, debido a la localización contigua al centro y el perfil turístico comercial. Al noroeste, Refinería sufre un impacto físico menor pero directamente vinculado con el desarrollo de Puerto Norte.

Las transformaciones físicas de la estructura parcelaria se producen de dos maneras. Por un lado, la rehabilitación de sitios patrimoniales en las cuatro áreas de protección histórica de Pichincha y edificaciones de carácter histórico de ambos barrios. Por otro lado, los procesos de 
completamiento en el interior de las áreas a través de obras de ampliación o reforma y la densificación del tejido localizado principalmente en los corredores urbanos. Estos procesos han sido significativamente superiores en Pichincha, particularmente entre los años 2005 y 2006 donde los indicadores urbanos eran favorables en todo el sector. En esos mismos años las demoliciones en el barrio fueron muy intensas, orientándose entre los años 2010 y 2014 hacia los corredores urbanos en ambos suburbios. Los dos casos involucran parcelas contiguas para grandes emprendimientos con características similares a Puerto Norte (tipologías, usos, destinatarios, etc.).

Los usos de suelo mantienen el perfil residencial y comienzan al mismo tiempo a reconvertir las antiguas instalaciones industriales de los sitios. Pichincha lo realiza primero para fines turísticos, comerciales y de negocios en toda su extensión mientras que Refinería solo orientado al comercio minorista en algunos sectores. Los corredores urbanos de uso mixto de suelo adquieren características particulares combinando las viviendas con nuevas actividades (Junín grandes concesionarias, Cándido Carballo pequeños comercios y Rivadavia - Del Valle entretenimiento nocturno). Igualmente el futuro desarrollo de estas dos últimas avenidas es incierto dado que tienen gran cantidad de lotes vacantes donde aún no se ha definido el uso final. Se estima que será más intensa la renovación en el corredor Cándido Carballo de Refinería.

\section{BIBLIOGRAFÍA}

BARENBOIM, Cintia Ariana. Políticas públicas urbanas y transformaciones edilicias: el caso del barrio histórico de Pichincha en la ciudad de Rosario, Argentina. Madrid: Editorial Académica Española, 2011.

BARENBOIM, Cintia Ariana. Evolución histórica de la costa central y su vinculación con el gran proyecto urbano de Puerto Norte en la ciudad de Rosario, Argentina. Revista Iberoamericana de Estudios Municipales, Santiago de Chile, N 10, p. 61-81, 2014.

CONSEJO MUNICIPAL. Ordenanza № 6.271 de Primer Fase del Centro de Renovación Urbana Scalabrini Ortiz: Sector Talleres. Rosario: Consejo Municipal Rosario, 1996.

CONSEJO MUNICIPAL. Ordenanza № 7.892 de la Segunda Fase del Centro de Renovación Urbana Raúl Scalabrini Ortiz: Plan Especial Puerto Norte. Rosario: Consejo Municipal Rosario, 2005.

CONSEJO MUNICIPAL. Ordenanza № 8.125 Plan Especial Barrio Pichincha. Rosario: Consejo Municipal Rosario, 2006.

CONSEJO MUNICIPAL. Ordenanza № 7.956 Plan Especial Av. de las Tres Vías Luis Cándido Carballo. Rosario: Consejo Municipal Rosario, 2010. 
CONSEJO MUNICIPAL. Ordenanza N $\cong 8.980$ de Reordenamiento Urbanístico Segundo Anillo Perimetral al Área Central, sector Refinería. Rosario: Consejo Municipal Rosario, 2012.

CUENYA, BEATRIZ. Grandes proyectos urbanos, cambios de centralidad urbana y conflicto de intereses. Notas sobre la experiencia argentina. En Cuenya, Beatriz. Novais, Pedro y Vainer Carlos (compiladores). Grandes Proyectos Urbanos. Miradas críticas sobre la experiencia argentina y brasilera. Buenos Aires: Café de las ciudades, 2012.

DIRECCIÓN GENERAL DEL PLAN DIRECTOR. Plan Urbano Rosario 2007 - 2017. Rosario: Secretaria de Planeamiento de la Municipalidad de Rosario, 2011.

DÓCOLA, Silvia. y MÚGICA, María Luisa. Miradas históricas y espaciales para el Plan Especial Pichincha de Rosario. IV Jornadas Nacionales Espacio, Memoria e Identidad. Rosario: Facultad de Humanidades y Artes, Facultad de Ciencias Políticas y Relaciones Internacionales de la UNR, 2006.

INSTITUTO NACIONAL DE ESTADÍSTICA Y CENSOS DE LA REPÚBLICA ARGENTINA. Censo Nacional de Población, Hogares y Vivienda 2001 y 2010. Ministerio de Económica: Buenos Aires, 2014.

LUNGO, Mario. Grandes proyectos urbanos. Una visión en general. En Grandes proyectos urbanos. El Salvador: Lincoln Institute of Land Police, 2004.

MARTíNEZ DE SAN VICENTE, Isabel. Plan Director Rosario. Estudio Particularizado del Área de atravesamiento Norte. Cuadernos del CURDIUR, Rosario, No 33. Rosario: Facultad de Arquitectura, Planeamiento y Diseño, UNR, 1987.

Trabalho enviado em 21 de agosto de 2015.

Aceito em 03 de setembro de 2015. 